\title{
The Virtual Collaborative Organization: Magnifying Transcultural, Technological and Strategic Complexity
}

\author{
Shauna Torrance, Christine Anne Reilly and Amos P. Haniff*
}

Heriot-Watt University, Edinburgh, EH14 4AS, UK

\begin{abstract}
This paper introduces the concept of the Virtual Collaborative Organisation (VCO), as an evolution from the single, temporary organisation. A VCO is described as a structure where multiple organisations enter strategic partnerships through a collaborative virtual project. The paper undertakes a critique of existing literature on temporary organisations to examine the complexity of achieving strategic alignment within a multiple organisation virtual collaboration. Themes arising from the management of such organisations are discussed in detail, before the role of technology in negotiating strategic alignment within and across multiple organisations is considered. Finally, tensions arising from alignment are debated, and the conclusion is drawn, while the VCO offers organisations a useful route to successful collaboration, tensions remain in the quest for strategic alignment.
\end{abstract}

Keywords: Collaboration, project management, projects, strategic alignment, temporary organisations, virtual organisations.

\section{INTRODUCTION}

The growing importance of project and project management practices in driving forward the competitive strategy of permanent parent organisations has resulted in the project being recognised as a temporary organisation $[1,2]$. The growth of projects and project management suggests such methods have become efficient vehicles for achieving organisational objectives within continually transforming environments [3-6]. Thus, the project has become more than a tool for implementation and production in accounting for further complexity, diversity and technicality in application, control and strategy beyond the traditions of time, cost and quality considerations $[7,8]$. The fast-paced growth of global markets and technological advancement has led to evolution of these temporary organisational forms into the virtual environment $[7$, $9,10]$. This paper introduces the concept of the 'Virtual Collaborative Organisation (VCO), which has developed from the existing concept of the single temporary organisation, arising as a result of the growing competitive importance of strategic partnerships and joint ventures between multiple organisations operating through the virtual sphere.

Central to this discussion is the maintenance of strategic alignment in negotiating and communicating the correlation and fit between permanent and temporary organisational strategy throughout the project life cycle, which is vital in achieving future competitive goals [3, 4]. Moreover, market conditions perpetuating cost focus, market knowledge and competitive advantage have led to collaborative trends in inter-organisational relationships managed through a single, temporary project $[11,12]$.

*Address correspondence to this author at the Heriot-Watt University, Edinburgh, EH14 4AS, UK; Tel: +44(0)131 451 3847;

E-mail: a.haniff@hw.ac.uk
While acknowledging the pivotal role technology has played in linking business, organisations and culture today, the codependency of technology, culture and communicat-ion across organisational and international boundaries has resulted in a plethora of challenges associated with operating on a temporary project and virtual basis $[9,13]$. It will, therefore, be suggested that in attempting to achieve project success and obtain strategic alignment between multiple organisations in this context, such challenges are significantly magnified in comparison to virtual projects operating in the simplest, temporary form.

By way of a significant critique of existing literature the paper will firstly seek to examine and assess the complexity of achieving strategic alignment within the context of a multiple virtual collaboration through a single, temporary project. Residing complexities and themes involved in managing these organisational forms will then be examined. This will include negotiation the lack of a single line of authority, collaboration across cultures and alignment of existing practices and bureaucracies across international and organisational borders. Finally, the vital role of technology used in collaboration and communication will be considered along with tension surrounding the need for standardisation within the VCO, before concluding with a summary of alignment tensions and areas of future research.

\section{BACKGROUND LITERATURE}

\section{Temporary Organisational Theory}

Projects have become more complex and important than traditional management theory indicates $[1,3,7]$. No longer is the coordination of project activities seen to be confined to the traditional process based themes of time, cost and quality, but may be considered multifaceted, social, emerging organisations flexible to change within the current turbulent business environment $[1,2,8]$. Increasing acknowledgement of these 
complexities has led to the acceptance of the project as a temporary organisation. It is apparent that while earlier definitions place emphasis on its temporary nature and predefined goals [2], later interpretations look to the social elements of the temporary organisation often defining it as a "set of organizational actors working together on a complex task over a limited period of time" [7, p. 468, 14]. As a consequence, definitions correlate more effectively with the intricate application of strategy, control, coordination and management employed. This simultaneously broadens their application to a variety of contexts including the $\mathrm{VCO}$, whereby multiple transcultural permanent organisations enter into strategic partnerships through the use of a single collaborative virtual project producing a multifaceted interface and network of relations.

Alternative research has focused on the varying interface between the permanent parent organisation and the temporary organisation including analysis surrounding permanency and time span [8], task-based capabilities [1], levels of dependency [12] and innovative abilities [15] in accounting for areas of tension in achieving strategic goals. Modig [16] makes a particularly unique and important contribution within this area. In concentrating on the role and interface between temporary and permanent organisations, the author develops a continuum upon which organisational forms may be placed to determine the unique contextual relations that take place and levels of reliance experienced. While the conceptual framework is based on only four large scale case studies, its ability to account for variety is important given the diversity of temporary projects being undertaken including forms such as the $\operatorname{VCO}[7,11,12]$.

While definitions and explanations surrounding theories of the project as a temporary organisation have become increasingly inclusive of a variety of forms and different industries, research providing solutions to tensions within the simplest relations between two forms of organisations remain slim [7]. Researchers such as Grabher [12] point to more social and cultural aspects of collaboration between temporary and permanent organisations. In providing rationales for development of more effective relations through identification of challenges inherent within organisational learning, trust and cultural conditioning in temporary collaborative arrangements, the author seems to imply repeated, long term collaboration may be a solution to such complexities. It is argued that this would result in sustained processes, understanding in collaboration, and the creation of trust throughout the temporary lives of different projects within a sector [12]. However, given the multitude of parent organisations contributing towards the single temporary organisation within the context of the VCO, it may be suggested the practicality of such repeated collaboration given the variety of organisational aims and goals, may be in doubt.

The interface between temporary and permanent organisations will inevitably affect the level of strategic alignment attained within a project, and therefore directly impact on the success of such projects. While this is an evidently difficult task to achieve under the authority and collaboration of a single parent and temporary organisation, the task becomes more arduous when considering the diluted authoritative control and leadership of multiple parent organisations within a VCO.

\section{Strategic Alignment}

There exists widespread support for the presupposition that strategic alignment of temporary organisational goals with that of the permanent organisation is vital to the continued success of projects $[3,4,6]$. In measuring success, there is a growing body of academic literature which seeks to distinguish between more objective factors contributing to project management success often located within the parameters of time, cost and quality and more subjective matters associated with project success including stakeholder management and review [17-20]. Subjective success factors become considerably more important when considering the large number of transcultural stakeholders tied into projects conducted through the VCO. As a result, discussion surrounding strategic alignment of these often differing needs becomes increasingly important.

Strategic alignment is most commonly illustrated through the hierarchical translation of strategy, and is depicted as the dynamic cascading of corporate objectives through the organisational structure, from corporate board level through to strategic business units and implementation at a functional project level $[6,21]$. Hierarchical models, like that employed by Archibald [22], indicate the multifaceted and inclusive nature of projects. This highlights the fact that they are never implemented in isolation to the wider organisational structure, goals and purpose [23]. Jamieson and Morris [21] point to the existence of both qualitative and quantitative evidence suggesting there are normative structures present within organisations to aid strategic alignment, thereby providing evidence attesting to its importance. However, such analysis, illustrations and solutions become increasingly irrelevant in the context of the VCO. Here, strategic alignment of corporate objectives is negotiated across the external organisational boundaries of different transcultural permanent organisations through the virtual means of a single project as opposed to the conventional project where alignment is often negotiated from within the boundaries of a single organisation.

Moreover, in focusing on the concept and variance of strategy arising within the turbulence of the modern business environment, complexity of achieving strategic alignment is further exacerbated $[4,24]$. The work of Mintzberg and Waters [25] remains seminal within this area. Through a large crossindustry study, the authors identified both deliberate and emerging strategy. The former pointing to the realisation of planned and intended strategy and the later denoting strategies realised without intention [25, p.258]. The conclusion that projects most often consist of a mixture of the two variances of strategy has significant implications for the ability of organisations to communicate and achieve strategic alignment. This has led to differences in views as to the realism of strategic alignment models. Mackay and Marshall [26] researching within the context of alignment of organisations and information systems have implied that the front-end precision quest for hierarchical strategic alignment is too reliant upon the realisation of deliberate strategy for project success and is, therefore, ultimately flawed. They argue that given the human element of temporary organisations and the chaotic nature of strategy within these environments, that realisation of strategy remains a contextual problem that depends on unique circumstances and actions rather than maintenance of broad applicability. 
In acknowledging the complexity of communicating and translating emergent organisational strategy through alignment of temporary and permanent organisational goals, a number of researchers have pointed to the effectiveness of viewing the process as a two-way relationship $[4,6,24]$ recognising the interrelated and inclusive nature of strategy formation [23]. In fact, Srivannaboon and Milosevic [24] further such arguments in referring to a process of mediation between deliberate, frontend strategy and emergent strategies, whereby the permanent organisation promotes two-way communication from both ends of the hierarchy in creating a culture of adaptation, responsiveness and innovation. This notion is also supported by Besner and Hobbes [27] who advocate the official formation of communication channels to aid the strategic mediation process.

While structures presented within research have proven effective and applicable within the conventional project setting, it may be argued when considering a structure consisting of multiple permanent, authoritative organisations, the process of mediation becomes further complicated. The two-way process of communication becomes multiplied and so harder to accomplish resulting in existing academic findings having less relevance within this context. Grabher [12] recognises that with inter-firm networks becoming an increasingly valid action within the business environment, "conceptions of firms as islands of hierarchical co-ordination...in mere caricatures" [12, p.205].

\section{The Virtual Collaborative Organisation: Magnifying Existing Challenges}

Recent advancements and innovations in communication technology have allowed collaboration between multiple permanent organisations. This takes place within the virtual sphere through a single temporary and virtual organisation, referred to within this paper as the 'Virtual Collaborative Organisation'. The increasing use of the virtual domain has, in fact, been associated with a growing number of benefits including the maximisation of knowledge and resources regardless of geographical location, cost effectiveness, flexibility and increased innovation [9, 10, 28-32].

While the role technology has played in realising innovative forms of value creation is evident, it has already been demonstrated that challenges associated with achieving strategic alignment and managing the interface between multiple permanent organisations by way of a single temporary project organisation are considerable and complex in nature. Hence, it remains important to acknowledge the variety of challenges associated with virtual implementation of a project, especially where it is executed on an international basis. There have been a large number of academic research studies conducted within this area which have identified and analysed a significant number of challenges [9, 30, 33-35]. Challenges documented range from consequences associated with lack of face-to-face communication $[9,13,33]$, to heavy reliance on technology [9] and management across time zones $[9,10]$ to name a few. However, it should be noted that this area cannot be characterised by consensus producing disagreement as to the advantages and disadvantages of all issues identified.

As already documented, in accepting the above challenges it will be argued that within the collaborative environment of the VCO, new challenges and problems may arise, and existing challenges are likely to be magnified. This may be attributed to the multitude of organisations claiming authoritative control, and seeking to achieve variable strategic goals. This part of the paper will, therefore, seek to further analyse these challenges in connection with the VCO in working towards explicit identification of unique challenges as well as areas of future research. The challenges of managing and negotiating through a diluted form of governance will first be analysed, followed by an examination of the impact inevitable clashing of competing stakeholder objectives has on communication and progress as well as the difficulties of managing across cultures. Finally the importance of developing robust technological arrangements will be considered.

\section{Negotiating Control and Leadership}

Within the context of the $\mathrm{VCO}$, it can be argued that governance is a key issue [11] given the fact that managers have been identified as an important factor determining project team effectiveness [36]. Indeed, researching within the confines of the more traditionally structured project, Cooke-Davies [19] observes that people are responsible for all stages of a project lifecycle and so become ultimately responsible for its success or failure. This therefore implies that governance and leadership is a key director and variant, determining project success as well as ensuring strategic alignment of corporate objectives. However, within the unique structure of the VCO the project manager can no longer be standardised as the "Chief Executive of the temporary organization" $[1, p .5]$ as such control is necessarily diluted as a direct consequence of seeking decentralised strategic, multiple collaboration [37]. As a result, the onus of seeking to achieve strategic alignment is no longer confined to single project management authority which in itself brings unique leadership challenges when operating within the virtual sphere [38]. Rather, leadership authority is divided among those governing the objectives and goals of each parent organisation through a single virtual project.

There is a substantial body of existing literature within the area of virtual projects, leadership and governance issues [11, $28,31,38,39]$. Whilst a tenuous consensus that the challenges arising within this area are particularly acute exists observations and analysis pinpointing appropriate leadership and governance techniques remains scattered. Cascio [31] links the importance of governance to managing and monitoring performance of virtual teams. It is argued that appropriate leaders should form and communicate clear expectations and create conditions in which they may be achieved and encourage progression and improvement. However, the author concedes that given current trends within the business environment, the importance and complexity of performing such skills are likely to escalate as new forms of virtual structures, including that of the VCO, come into existence.

Both, Cascio [31] and Malhotra et al. [38] agree on the continued applicability of effective management theories making the generalisation that leaders of all types of teams retain certain roles and qualities but become more important under certain circumstances. Malhotra et al. [38] identified a number of these leadership styles through quantitative and qualitative research ranging from establishment of trust and employee engagement to effective performance monitoring. The authors place emphasis on the universally applicable skills such as communication of goals, execution plans, structure, and vision, in governing such arrangements. The focus 
demonstrated here remains on leadership abilities in communication of execution plans and clarification of vision, although team roles are highlighted by Oerlemans and Pretorius [11] and Stevenson and McGrath [36]. However, research conducted by Oerlemans and Pretorius [11] specialising in interorganisational collaboration highlights the importance of using formal contracts in clarifying and confirming the interface between various organisations. The later comparative study highlights the importance of solid work structures governed by clear reporting procedures.

Other academic research places emphasis on the importance of adaptability and flexibility in leading and governing project teams $[28,36,39]$. It may be argued that findings of this kind may be readily transferrable to various temporary organisational forms including that of the VCO. Research conducted by Davis [28] is particularly relevant in rightly observing the dilution of leadership in virtual teams seeing influence as weakening across time and distance. Moreover, in employing the concept of 'Tao of leadership' within the virtual environment, the authors effectively imply appropriate leaders are legitimised through their ability to transfer and adapt their skills to the circumstances in which they represent governing authority. This proposition is supported by previous research carried out by Solomon [39] who recommends the rotation of leadership through the project life cycles through use of capable team members supported by rigorous training regimes.

Suggestions of effective communication skills and continuous clarification and provision of vision, project execution plans and team roles appear important to any project or temporary organisation including the VCO. Training emphasising adaptability and flexibility in dealing with the structure and project environment, including suggestions of rotated leadership may be vital to leadership and governance.

\section{Communicating Multiple Stakeholder Objectives}

Communication remains a central theme within the context of operating projects within the virtual sphere given the geographical dispersion of temporary organisational team members $[9,10,13,33,40]$ and the fact that collaboration necessarily requires communication and information exchange to function effectively [37]. The complexity demonstrated here is magnified by large numbers of permanent organisational stakeholders communicating within the operations of the VCO, each retaining equal authority to communicate vision and retain their heterogeneous forms [41]. It becomes inevitable within this organisational structure that each permanent parent organisation will have their own unique agenda and individual strategic objectives to achieve, resulting in communication via technology becoming vital to the strategic alignment of organisational goals and ultimately project success. Moreover it has been observed that the risk of communication breakdown between the multiple parties inevitably correlates to the increased needs of the project [42].

It may be observed that this unique structure is not dissimilar to the multi-project environments of the project based organisations and may, in fact, mirror its competitiveness in communicating and attempting to implement individual permanent organisational priorities. Haniff and Fernie [3] have observed a collision of strategies within these competitive organisational environments. In competing for scarce resources, each project team is isolated from the whole in ensuring their individual strategy is strategically aligned to that of the organisation. This produces boundaries and alliances between project teams operating within the same environment. It is argued here that under such circumstances project aims and goals become disparate and fragmented, not fully acknowledging the vision and goals of the permanent organisation as a whole. While the authors refer to competition for resources to support implementation, within the VCO competition may be located in gaining authority of communication over other collaborating permanent parent organisations to push forward as well as encourage individual agendas and ensure individual strategic alignment.

It is generally agreed that training of virtual team members is vital to effective communication throughout the lifecycle of the project enabling members to communicate through and adapt to the organisational and technological constraints of working within the virtual environment [33]. However, in considering the participatory nature of the virtual global team, especially in the context of the VCO, the notion of team membership and trust also becomes important. A number of researchers have argued that lack of face-to-face communication and a sense of shared membership has had an adverse effect on the creation of trust needed within such collaborative innovative environments like the VCO $[9,10,34$, $35]$. Furthermore, there is general agreement that physical, faceto-face, forms of communication are important in fostering trust in a turbulent environment where a high level of control and knowledge is required [9, 10, 13, 31]. Nevertheless, Cascio [31] perpetuates the creation of trust through more interactive and informal processes of maintaining positive attitudes and communication through more informal means and promoting a clear understanding of roles. Meyerson et al. [14] employ the notion of 'swift trust' created within temporary organisational environments limited by time, whereby the team is focused on a common task. It is implied that trust is fostered within striving towards a mutual strategy through focus and enthusiasm for success as opposed to focus on interpersonal social cues and relationships found in face-to-face communication.

While the research and theoretical argument posed by Meyerson et al. [14] could apply to the VCO, in that the project team is centred on a common task, the proposition that the strategies of the multiple parent organisations are identical is extremely unrealistic placing the applicability of the concept of swift trust in doubt. However, it remains clear that for vital communication to be effective, creation of trust between collaborative permanent organisations is needed. The key issue, therefore, is achieving trust and cooperation in locating a form of communication which enables parties to work holistically in achieving individual and general strategic alignment. Only with this will virtual projects operating within the VCO to become successful.

\section{Overcoming Cultural and Functional Diversity}

The growing trend towards inter-organisational collaboration to achieve competitive advantage and increased innovations has also resulted in creation of culturally diverse virtual teams chosen due to specialism and expertise [10, 13, 42, 43]. The challenges associated with communicating across cultural backgrounds are widely recognised within current research, which often distinguishes between tensions created by 
international and functional organisational culture [13]. Moreover, in focusing on both international and functional organisational culture, it is argued here that given the diverse governance inherent within the VCO, the tensions created in communicating and combining the expertise of different cultures seems inevitable. Despite willingness to collaborate, there are challenges in communicating across different cultural customs and organisational norms, as well as the need to negotiate a collaborative platform able to overlap the functional diversity of each permanent parent organisation to allow the VCO to function effectively and achieve success in attaining strategic alignment across multiple organisational visions. While the VCO, as a temporary organisation, is intended to rise above the bureaucracy and routine of each permanent organisation, the challenges and conflicts arising in doing so become increasingly more complex given that coordination must also be achieved.

Challenges arising in intercultural collaboration therefore become particularly important in maintaining alignment of the multiple organisational objectives through the VCO. It has been repeatedly highlighted that differences in culture have the potential to cause communication breakdown, considered a vital factor in ensuring the success of virtual projects of any form [42]. There is an existing consensus that virtual organisational forms challenge traditional assumptions made about organisational conceptions of culture in failing to exhibit stable, fluid and shared perceptions, assumptions and values [42, 43]. Rather, organisational forms such as the VCO face continual and unique tensions at the interface of organisational communication including language barriers as well as alternative perceptions and understandings [13]. All factors combined making it difficult to achieve common ground needed for effective teamwork to occur [42].

Related to this, Oertig and Buergi's [10] investigation into challenges faced by virtual project team leaders pointed to language barriers and cultural attitudes originating from international affiliation becoming a key challenge and tension within such environments. This is ultimately affecting decision making on team recruitment, communication and trust. Qualitative interviews conducted indicated issues arose in relation to cultural interpretation of tone of speech, differences in tensions communicated and distrust of motives. Daim et al. [13] confirm the importance and implications of such findings in denoting the impact different cultural expectations and understandings can have on team performance arguing national cultures often vary in views of effectiveness of individualism or collectivism within teams, directly affecting development of interpersonal relationships and team dynamics.

However, combining multiple organisational cultures and functional structures present equally, if not more, of a challenge to the strategic alignment of projects conducted within the context of the VCO. Bjørn and Ngwenyama [42] make a key contribution to this argument in distinguishing between a more general 'lifeworld' environment containing background, knowledge and assumptions, and the organisation and individual work process within project teams. In making this distinction it is argued that breakdown in communication occurs within individual work process levels requiring action at all levels to create a shared meaning vital to virtual teamwork and translucent communication protocols. This corresponds to suggestions posed by Wiesenfeld et al. [44] that organisational identification presents a key factor in facilitating the level of communication needed for virtual teams to function. While the authors point to use of technology to produce the coordination and control needed within diverse virtual teams such as the VCO. They also advocate increased frequency in communication to aid the creation of shared meanings, interpretations and assumptions. This, they argue, simultaneously creates ties and affiliations to the new structure and form of the organisation as advocated by Bjørn and Ngwenyama [42]. However, given the variety of functional organisational cultures operating within a $\mathrm{VCO}$, this task may be time consuming and arduous.

In accepting the challenge of creating shared meaning across multiple international and organisational cultures operating within the $\mathrm{VCO}$, the more holistic and flexible alternative analogy surrounding cultural tension presented by Gibbs [43] may become relevant. In employing the concept of the 'cultural kaleidoscope', the author identifies an error located within current literature and research in identifying culture as opposite to virtual organisational structures preferring to view combined cultures as exhibiting 'dynamic tensions'. It was therefore concluded that efforts to minimise differences are essentially flawed and that strategies, instead, create healthy tension through intercultural collaboration may become more successful by capturing diversity within a conceptual kaleidoscope. Thus this method is being able to account for multiple cultural dimensions from national, organisational and individual views and attitudes. By viewing the tension as fragmented and lacking consensus the framework becomes flexible for use within unique organisational forms like the $\mathrm{VCO}$ and aids in identifying, unifying and disaggregating features of the collaborative organisation without producing assumptions.

\section{Technological Complexity and Coordination}

It is undoubted that advancement and innovation surrounding communication technology has played a pivotal role in collaboration of organisations to maintain efficiencies, cost reduction, market focus and the leveraging of expertise beyond the confines of traditional external organisational boundaries [9, 10, 31, 45, 46]. Growth in commercial use of communication technology has empowered organisations to form virtual communal platforms in which they are able to communicate, coordinate and control collaborative efforts to facilitate strategic alignment of competitive objectives by way of virtual, temporary organisational forms [13, 33, 44, 46]. However, the increasing complexity of virtual organisational forms which evolve into collaborative efforts through single project environments such as the VCO have led to use of technology becoming a key issue and challenge. Again, the number of authoritative permanent organisations governing the virtual project by way of the VCO means typically there is no common technological platform on which to base communications, control and coordination. This could have serious adverse effects on alignment of projects against various unique corporate objectives involved.

However, the importance of technology in facilitating achievement of advanced, technically complex and collaborative strategic objectives cannot be underestimated. It is technology that provides an adaptive network for project management support and communication, vital to both strategic alignment and project success [33, 34, 47]. Aptly labelled 
'eCollaboration systems' [46, p. 181] such technological platforms have been heralded as organisational glue enabling collaborative virtual projects teams to gain some form of organisational identity and shared meaning in communication and coordination, the importance of which is discussed in the preceding section $[44$, p. 788$)$.

Through the provision of a selection of technological solutions, organisations have an array of choice in facilitating appropriate organisational fit and alignment with strategic aims [13, 44]. Zigurs et al. [33] and Reimer et al. [46] are particularly poignant in pointing to both benefits and availability of collaborative technology providing the interface between different organisations that could become potentially beneficial in the multifaceted environment of the VCO. The authors indicate more flexible forms of technology such as group workflow calendars, tasks, lists and plans as well as social networking interfaces via wikis, electronic whiteboards, video conferencing, communication devices and techniques add maximum benefit. They suggest each allows for ownership and shared authorship, helpful in developing shared meaning of communication within these environments.

On the other hand, in considering the multiplicity of strategic partners involved within the VCO, choosing a communication technology platform which allows for project control and coordination while simultaneously aligning with each individual organisation's current functions appears increasingly unrealistic. While some have focused on the ability of such technology to create a sense of ownership and collectively contribute to the virtual project, others have depicted collaborative technology as a "field in motion" both on a managerial and technological level. This, ultimately leading to uncertainty in application and unknown challenges in practice, which inevitably results in potential communication and alignment problems [46, p. 182]. Despite recent advances in collaborative technology, which is better able to facilitate the use of a communicative and community based approach, a plethora of empirical investigations show evidence that more simplistic email technique continues to prevail $[9,13,33]$, suggesting notions of shared vision and communication currently remain unrealised ambitions.

In focusing on the working environment in which the $\mathrm{VCO}$ sits, it seems particularly important to acknowledge that while collaborative communication technologies are developed to achieve certain tasks and work in set ways, the usefulness and applicability of these technological communication platforms ultimately depends upon the context in which they are used [9, $13,44,46]$. It may be argued here that technology evolves with the social contexts of organisations that place unique interpretations of best practice and procedure upon its use, presupposing that features are enabled and which techniques remain redundant. It is, therefore, valid to suggest that within the context of the VCO, which evidently operates on a tenuous and rather fragmented interface between multiple strategic partners, the use of collaborative technology to communicate, coordinate and control may become problematic given the number of interpretations which may be placed upon its use. This combined with the fact that the technological platform chosen is expected to align with the project as well as each governing permanent organisation's internal functions makes the task of achieving strategic alignment within the VCO extremely fragile.

\section{CONCLUSION}

This paper introduces the concept of the "Virtual Collaborative Organisation (VCO). This concept has developed from an existing model of the single temporary organisation, as a result of the growing competitive importance of strategic partnerships and joint ventures between multiple organisations operating through the virtual sphere. While the VCO provides opportunities for successful projects and project management practices, there are a number of challenges involved with negotiating within and across organisations in the virtual sphere.

An important feature of the VCO is the ability for strategic alignment to be negotiated across, rather than within, organisational boundaries. As the success factors are subjective within such an organisation, it is crucial that all parties involved negotiate strategic alignment. The success of a VCO is dependent upon flexibility, adaptability and shared leadership, as well as the key issue of flexible and collaborative use of technology.

Notwithstanding the potential for project success, the VCO raises a number of challenges for strategic alignment within and across the temporary and permanent organisations involved in collaboration. This can impact on overall project success, as well as ongoing relationships between participants. Mediation between participants can be complicated, and there is a risk of communication breakdown, particularly where shared communication processes have not been successfully designed. The level of communication needed to make the project successful, may be arduous, and the traditional challenges of intercultural collaboration are also present.

Further research and development within this area may find some use in focusing on how tensions in functional diversity might be resolved and whether collaborative technological platforms can be designed to support effective communication in virtual collaborative organisations. Finally it is suggested that without development in these areas, challenges will continue to exist in striving to strategically align multiple corporate objectives within virtual collaborative projects.

\section{CONFLICT OF INTEREST}

The authors confirm that this article content has no conflict of interest.

\section{ACKNOWLEDGEMENTS}

Declared none.

\section{REFERENCES}

[1] Turner JR, Müller R. On the nature of a project as a temporary organization. Int J Proj Manage 2003; 21: 1-8.

[2] Packendorff J. Inquiring into the temporary organisation: new directions for project management research. Scand J Manag 1995; 11: 319-33.

[3] Haniff AP, Fernie S. Projects: where strategies collide: Proceedings of CIB 2008 Joint CIB WO65/WO55 Symposium: Transformation through Construction, Dubai 2008.

[4] Srivannaboon S. Linking Project Management with Business Strategy. Project Management Institute 2006.

[5] Dietrich P, Lehtonen PI. Successful management of strategic intentions through multiple projects - Reflections from empirical study. Int J Proj Manage 2005; 23: 386-91.

[6] Morris PWG, Jamieson A. Moving from corporate strategy to project strategy. Proj Manage 2005; 36: 5-18. 
[7] Bakker RM. Taking stock of temporary organizational forms: a systematic review and research agenda. Int J Manag Rev 2010; 12: 46686.

[8] Lundin RA, Söderholm A. A theory of the temporary organization. Scand J Manag 1995; 11: 437-55.

[9] Lee-Kelley L, Sankey T. Global virtual teams for value creation and project success: A Case Study. Int J Proj Manage 2008; 26: 51-62.

[10] Oertig M, Buergi T. The challenges of managing cross-cultural virtual project teams. Team Perform Manag 2006; 12: 23-30.

[11] Oerlemans L, Pretorius T. Governing temporary interorganisational projects. Innovate $2010 ; 4: 31-5$.

[12] Grabher G. Cool projects, boring institutions: temporary collaboration in social context. Regional Stud 2010; 36: 205-14.

[13] Daim TU, Ha A, Reutiman S, et al. Exploring communication breakdown in global virtual teams. Int J Proj Manage 2012; 30: 199212.

[14] Meyerson D, Weick KE, Kramer RM. Swift trust and temporary groups. In: Kramer RM, Tyler TR, Eds. Trust in Organizations: Frontiers of Theory and Research, Sage: Thousand Oaks, CA 1996; pp. 166-95.

[15] Engwall M, Svensson C. Cheetah teams in product development: the most extreme form of temporary organization? Scand J Manag 2004; 20: $297-317$

[16] Modig N. A continuum of organizations formed to carry out projects: Temporary and stationary organization forms. Int J Proj Manage 2007; 25: 807-14

[17] Hughes SW, Tippett DD, Thomas WK. Measuring project success in the construction industry. Eng Manage J 2004; 16: 31-7.

[18] Collins A, Baccarini D. Project success - a survey. J Constr Res 2004; 5:211-31.

[19] Cooke-Davies T. The "real" success factors on projects. Int J Proj Manage 2002; 20: 185-90.

[20] Baccarini D. The logical framework method for defining project success. Proj Manage J 1999; 30: 25-32.

[21] Jamieson A, Morris PWG. Moving from corporate strategy to project strategy. In: Morris PWG, Pinto JK, Eds. The Wiley guide to project, program and portfolio management. Wiley: Hoboken, NJ 2007.

[22] Archibald R. Managing high-technology programs and projects. $3^{\text {rd }}$ ed. Wiley 2003.

[23] Cleland DI. Strategic Management: The Project Linkages. In: Morris PWG, Pinto JK, Eds. The Wiley guide to project, program and portfolio management. Wiley: Hoboken, NJ 2007.

[24] Srivannaboon S, Milosevic DZ. A two-way influence between business strategy and project management. Int J Proj Manage 2006; 24: 493-505.

[25] Mintzberg H, Waters AW. Of strategies deliberate and emergent. Strategic Manage J 1985; 6: 257-72.

[26] McKay J, Marshall P. 2 × $6=12$ or does it equal action research. $10^{\text {th }}$ Proceedings of the Australasian Conference on Information Systems 1999; pp. 597-609.

[27] Besner C, Hobbes B. The Perceived Value and Potential Contribution of Project Management Practices to Project Success. Proj Manage J 2006; 37: $37-48$
[28] Davis DD. The tao of leadership in virtual teams. Organ Dyn 2004; 33 : 47-62.

[29] Massey AP, Montoya-Weiss M, Hung Y. Because time matters: temporal coordination in global virtual project teams. J Manage Inform Syst 2003; 19: 129-55.

[30] Johnson P, Heimann V, O'Neill K. The "wonderland" of virtual teams. J Workplace Learn 2001; 13: 24-30.

[31] Cascio WF. Managing a virtual workplace. Acad Manage Exec 2000; 14: 81-90.

[32] McDermott L, Waite B, Brawley N. Putting together a world-class team. Training Develop 1999; 53: 46-51.

[33] Zigurs I, Khazanchi D, Memetjanov A. The Practice and Promise of Virtual Project Management. Virtual Technologies. Encyclopedia of ECollaboration 2008. IGI Global 2008: pp. 472-8.

[34] Kirkman BL, Rosen B, Gibson CB, Tesluk PE, McPherson SO. Five challenges to virtual team success: Lessons from Sabre, Inc. Acad Manage Exec 2002; 16: 67-79.

[35] Maznevski ML, Chudoba KM. Bridging space over time: global virtual teams dynamics and effectiveness. Organ Sci 2000; 11: 473-92.

[36] Stevenson W, McGrath EW. Differences between on-site and off-site teams: managers' perceptions. Team Perform Manag 2004; 10: 127-32.

[37] Kankaanpää T, Shamsuzzoha A, Carneiro L, et al. Methodology for non-hierarchical collaboration networks for complex products and manufacturing. Proceedings of the $16^{\text {th }}$ International Conference on Concurrent Enterprises. Switzerland 2010.

[38] Malhotra A, Majchrzak A, Rosen B. Leading virtual teams. Acad Manag Perspect 2007; 21: 60-70.

[39] Solomon C. Building teams across borders. Global Workforce 1998; 3 : 12-7.

[40] Pokharel S. Stakeholders' role in virtual project environment: a case study. J Eng Technol Manage 2011; 28: 201-14.

[41] Romero D, Galeano N, Molina A. Mechanisms for assessing and enhancing organisations' readiness for collaboration and collaborative networks. Int J Prod Res 2009; 47: 4691-710.

[42] Byørn P, Ngwenyama O. Virtual team collaboration: building share meaning, resolving breakdowns and creating translucence. Inf Syst 2009; 19: 227-53

[43] Gibbs JL. Culture as Kaleidoscope: Navigating Cultural Tensions in Global Collaboration. Proceedings of the ACM 2009 International Workshop on Intercultural Collaboration. Palo Alto, CA 2009.

[44] Wiesenfeld BM, Raghuram S, Garud R. Communication patterns as determinants of organisational identification in a virtual organization. Organ Sci 1999; 10.: 777-90.

[45] Westphal I, Thoben K, Seifert M. Managing collaboration performance to govern virtual organizations. J Intell Manuf 2010; 21: 311-20.

[46] Reimer K, Steinfield C, Vogel D. eCollaboration: On the nature and emergence of communication and collaboration technologies. Electron Mark 2009; 19: 181-8.

[47] Prasad K, Akhilesh KB. Global virtual teams: what impacts their design and performance? Team Perform Manag 2002; 8: 102-12. 